\title{
Effectiveness of Mobile Health Applications for 5\% Body Weight Reduction in Obese and Overweight Adults
}

\author{
Myeunghee Han', Sang Youl Rhee 2,* \\ 'Department of Nursing, Dongyang University, Yeongju; ${ }^{2}$ Department of Endocrinology and Metabolism, Kyung Hee University School of Medicine, Seoul, Korea
}

Background: World Health Organization reports that over 1.9 billion adults are obese. Studies have found that people who reduce their body weight by $5 \%$ experience considerable health benefits. Currently, mobile health (mHealth) applications (apps) show effectiveness in body weight reduction. The present study aimed to explore the effectiveness of a popular mHealth app in $5 \%$ body weight reduction and to identify factors that affect $5 \%$ body weight reduction in obese adults. We investigated the time it took users to achieve $5 \%$ body weight reduction according to usage characteristics and factors influencing this period of time.

Methods: This study was a secondary data analysis using data from 23,682 commercial mHealth app users. For analysis, logistic regressions, Kaplan-Meier estimators, log-rank tests, and Cox regressions were used.

Results: Variables in user characteristics including age (odds ratio [OR], 0.976; $P<0.001)$, male $(\mathrm{OR}, 1.226$; $P<0.001)$, initial body mass index $(\mathrm{OR}, 1.009 ; P<0.001)$, frequency of data entry for body weight $(\mathrm{OR}, 1.004$; $P<0.001)$, frequency of exercise (OR, 1.002; $P<0.001)$, and dinner intake $(\mathrm{OR}, 1.004 ; P<0.001)$ made significant contributions in predicting $5 \%$ weight reduction in the study cohort. Users who were obese and who more frequently entered their body weight, exercise, and dietary intake data reduced $5 \%$ body weight much sooner than other users. Data entry regarding initial body weight (exponentiation of the $B$ coefficient $[\operatorname{Exp}(B)], 1.002$; $P<0.001)$, frequency in body weight entry $(\operatorname{Exp}(B), 1,001 ; P<0.001)$, dinner intake $(\operatorname{Exp}(B), 1.003 ; P<0.001)$, and evening snack intake $(\operatorname{Exp}(B), 1.001 ; P<0.001)$ significantly contributed to predicting the time needed to achieve a $5 \%$ body weight reduction in users.

Conclusion: For $5 \%$ body weight reduction, mHealth apps are promising tools. Users who frequently monitor their health-related behaviors can expect a $5 \%$ reduction in body weight in a short period of time.

Key words: Telemedicine, Obesity, Body weight, Time, Weight loss, Overweight
Received February 16, 2021

Reviewed August 26, 2021

Accepted September 12, 2021

${ }^{*}$ Corresponding author

Sang Youl Rhee

https://orcid.org/0000-0003-0119-5818

Department of Endocrinology and Metabolism, Kyung Hee University School of Medicine, 26 Kyungheedae-ro, Dongdaemun-gu, Seoul 02447, Korea

Tel: +82-2-958-8200

Fax: +82-2-968-1848

E-mail:rheesy@khu.ac.kr

\section{INTRODUCTION}

According to the World Health Organization (WHO), as of 2016, over 1.9 billion adults aged 18 years and older were overweight, and over 650 million of these adults were obese. ${ }^{1}$ People who are overweight or obese are at increased risk for chronic diseases such as heart disease, stroke, hypertension, diabetes, and cancer. ${ }^{2}$ According to Gorin, ${ }^{3}$ people who lose between 5\% and 10\% of body weight will benefit from increased high-density lipoprotein cholesterol and decreased triglycerides. Similarly, the Centers for Disease Control and Prevention (2018) reports that people who reduce their total body weight by $5 \%-10 \%$, regardless of their initial body weight, are likely to obtain positive health outcomes such as improvements in blood pressure, cholesterol, and glucose levels. ${ }^{4}$

Nevertheless, achievement of $5 \%$ body weight reduction and the long-term maintenance of reduced body weight are difficult tasks

Copyright (c) 2021 Korean Society for the Study of Obesity

(ब) This is an Open Access article distributed under the terms of the Creative Commons Attribution Non-Commercial License (https://creativecommons.org/licenses/by-nc/4.0/) which permits unrestricted non-commercial use, distribution, and reproduction in any medium, provided the original work is properly cited. 
for overweight or obese people — such loss is associated with a high risk of failure and body weight regain. ${ }^{5}$ Previous studies have found that several factors are associated with better body weight loss and maintenance. Such factors include high levels of physical activity, low-calorie and low-fat dietary intake, and frequency in body weight self-monitoring. ${ }^{6}$ Accordingly, effective interventions that consider these factors are needed to support body weight reduction. ${ }^{7}$

At present, mobile health (mHealth) applications (apps) have been highlighted as effective tools for body weight management. Such $\mathrm{mHealth}$ apps provide sufficient information on body weight management and the success of specific eating and physical activity behaviors for body weight loss. ${ }^{8-10}$ In addition, mHealth apps offer opportunities for the self-monitoring of body weight, physical activity, and diet, all of which are the most influential factors in body weight reduction. Many previous studies have demonstrated that mHealth app-based interventions are acceptable and effective in promoting body weight loss in overweight and obese individuals. ${ }^{11}$ However, a lower effectiveness in achieving 5\%-10\% body weight reduction has been reported among overweight or obese adults. ${ }^{11}$ In addition, information about the time needed to achieve a 5\% body weight reduction among mHealth app users is scarce. Similarly, little is known about the factors that influence this period of time.

In this context, the present study aimed to examine the effectiveness of a popular mHealth app in 5\% body weight reduction in overweight or obese users. Specifically, we set the following objectives: (1) to explore differences in characteristics between 5\% and non$5 \%$ body weight reduction groups, (2) to identify factors influencing $5 \%$ body weight reduction, (3) to assess differences in the time needed to achieve $5 \%$ body weight reduction according to the characteristics of app users, and (4) to establish factors related to the time needed to achieve $5 \%$ body weight reduction among the study cohort.

\section{METHODS}

\section{Participants and study design}

This retrospective cohort study was conducted using data obtained from users of the fitness and weight loss app Noom Coach for 6 months between October 2012 and April 2014. Data included demographic characteristics, exercise habits, dietary intake, and body weight entered by users. A total of 48,095 users engaged with the app during our period of analysis. Users who were 42 years old $(n=4,041,8.40 \%)$, however, were excluded from the dataset because 42 years is the default age value of the app, and we assumed that many users did not change the age upon initial use. Furthermore, we also excluded users who were underweight or had normal weight $(n=13,037)$, as well as users with no data or with error records $(n=6,103)$. In addition, users under 20 years of age $(n=1,232)$ were excluded. The final number of users was 23,682 .

\section{Ethics statement}

This study was conducted in accordance with guidelines from the Declaration of Helsinki and the privacy policies of Noom Inc., and was approved by the Kyung Hee University Hospital Institutional Review Board (IRB No. KMC IRB 1435-04), which confirmed the absence of risk for any leakage of de-identified personal information. The need for informed consent from subjects was waived by the KMC IRB due to this study's retrospective design.

\section{Intervention: Noom Coach}

Noom Coach (Noom Inc., New York, NY, USA) is a commercially available mHealth app. This app has functions of tracking dietary intake, physical activity, and body weight. This app is published in five different languages and has been downloaded worldwide for purposes of health promotion. Since 2012, Noom Coach has been downloaded from the Google Play store and used by over 10 million people worldwide. In addition, the app has been consistently ranked as the most effective app for body weight loss. ${ }^{12,13}$ When users $\log$ in for the first time, they enter their goals for desired body weight and are prompted to record their current body weight and height. Throughout usage of this app, users are asked to record their dietary intake and number of steps daily. Based on the data entered by users, the app reports trends in body weight changes, calorie intake, and nutritional summaries. Noom Coach also provides customized feedback to support users' achievement of desired body weight. ${ }^{13,14}$

\section{Variables}

All data were obtained from app records. Data for body weight, dietary intake (breakfast, morning snack, lunch, afternoon snack, 
dinner, and evening snack), and exercise habits were self-reported and entered daily by app users.

\section{Demographic factors}

Included in our analysis herein are user demographic factors of age (years), sex (female or male), initial body weight (kg), body mass index (BMI; $\mathrm{kg} / \mathrm{m}^{2}$, overweight or obese), user location (Africa, America, Asia, Europe, or Australia), and version of the app (free or paid subscription version). BMI was calculated by the formula of body weight in kilograms ( $\mathrm{kg}$ ) divided by height squared in meters. Being overweight was categorized with BMI values between 25.0 and 30.0, while users with BMI 30 or higher were categorized as obese. ${ }^{15,16}$

\section{Self-monitoring factors}

Self-monitoring factors used in the present analysis included body weight, baseline calories ( $\mathrm{kcal}$ ), and total number of data entries for breakfast, morning snack, lunch, afternoon snack, dinner, and evening snack for 6 months. To obtain the total number of data entries during the 6 months for each category, we counted the total number of days that users entered their data.

\section{Time required to achieve $5 \%$ body weight reduction}

The primary outcome variable was the number of days that users needed to achieve $5 \%$ body weight reduction from when they first started using the app to 6 months.

\section{Statistical methods}

All analyses were conducted using IBM SPSS version 24 (IBM Corp., Armonk, NY, USA). Prior to data analysis, exploratory data analysis was conducted to identify errors in coding, examine missing data, and check normality. All assumptions for specific analysis were checked to accurately conduct the analysis. If a subject's body weight on a particular date was missing, the value was replaced with the average body weight calculated by averaging the body weight pre- and post-seven days from the date.

To evaluate the difference between 5\% and non-5\% body weight reduction groups, independent t-tests and chi-square tests were used. The $5 \%$ body weight reduction group included users who reduced their body weight by $5 \%$ from their original body weight within 6 months. Users with any outcomes otherwise were included in the non-5\% body weight reduction group. In addition, logistic regression was conducted to identify factors influencing the likelihood of 5\% body weight reduction. For logistic regression, univariate logistic regression models were conducted on a selection of significant variables at a significance level of 0.20 . After entering all significant variables in the logistic model, backward elimination was conducted at a significance level of 0.05 until all predictors achieved statistical significance.

To identify time differences in 5\% body weight reduction according to demographic and self-monitoring factors, Kaplan-Meier (K-M) estimators and log-rank tests were performed. For K-M estimators, continuous variables were categorized into two groups based on their average value. For example, if the frequency of exercise data entry was under the average value, then the corresponding users were included in the low group. Alternatively, if users showed frequency of exercise data entry over the average value, then they were assigned to the high group.

Cox hazard regression models were used to evaluate the impact of the different variables that related to the time required to achieve $5 \%$ reduction in body weight. In this model, users who achieved the $5 \%$ body weight reduction were codified with "event (1)", while those who failed to reduce their body weight by $5 \%$ were codified with "failure (0)". Time was defined as the time required to achieve $5 \%$ body weight reduction within 6 months from initial app usage. Covariates included age, initial body weight, initial BMI, gender, location, baseline calorie expenditure due to exercise, dietary intake for breakfast, morning snack, lunch, afternoon snack, dinner, and evening snack, as well as the total frequency of data entry for body weight, exercise, breakfast, morning snack, lunch, afternoon snack, dinner, and evening snack. Using backward elimination, the final model was determined. Statistical significance was assumed when $P$-values (two-sided) were below 0.05 .

\section{RESULTS}

\section{Demographic characteristics of users}

A total of 23,682 users were included in the dataset. The average age of users was 36.55 years (standard deviation, 11.00 years). Over half of the users $(n=16,307,68.86 \%)$ were female. Overweight 
Table 1. Demographic characteristics $(n=23,682)$

\begin{tabular}{lc}
\hline Characteristics & Value \\
\hline Age $(\mathrm{yr})$ & $36.55 \pm 11.00(20-80)$ \\
Sex & $7,375(31.14)$ \\
Male & $16,307(68.86)$ \\
Female & \\
Body mass index $\left(\mathrm{kg} / \mathrm{m}^{2}\right)$ & $11,690(49.36)$ \\
Overweight & $11,992(50.64)$ \\
Obese & $90.54 \pm 19.27(44.00-229.97)$ \\
Body weight $(\mathrm{kg})$ & \\
Location* & $9,609(40.62)$ \\
Europe & $8,943(37.80)$ \\
America & $4,012(16.93)$ \\
Asia & $624(2.64)$ \\
Australia & $470(1.99)$ \\
Africa & \\
App version & $22,067(93.18)$ \\
Free trial & $1,615(6.82)$ \\
Paid version &
\end{tabular}

Values are presented as mean \pm standard deviation (range) or number $(\%)$.

*Some values missing.

and obese users totaled 11,691 (49.36\%) and 11,992 (50.64\%), respectively. The initial body weight of users was $90.54 \pm 19.27 \mathrm{~kg}$. Most users were from Europe $(n=9,609,40.62 \%)$ and America $(n=8,943,37.80 \%)$, and most of them $(n=22,067,93.18 \%)$ used the free version of the app (Table 1).

\section{Characteristics of users' self-monitoring}

For 6 months, the average calories of exercise, breakfast, morning snack, lunch, afternoon, dinner, and evening snack were 292.97, $278.10,162.92,399.63,195.57,454.43$, and 197.54, respectively. Averages for total number of data entries for body weight and exercise for 6 months amounted to 76.50 and 63.11, respectively. On average, users entered data for body weight, exercise, breakfast, morning snack, lunch, afternoon snack, dinner, and evening snack $118.37,44.75,106.80,53.66,89.62$, and 28.23 times for 6 months, respectively (Table 2 ).

\section{Differences in demographic and self-monitoring factors} between $5 \%$ and non-5\% body weight reduction groups

We observed significant differences between the 5\% and non-5\% body weight reduction groups in terms of demographic factors, average dietary intake calories, and total number of data entries dur-
Table 2. Self-monitoring characteristics $(n=23,682$

\begin{tabular}{lc}
\hline Category & Mean \pm SD (range) \\
\hline Calorie average & \\
Exercise & $292.97 \pm 207.41(0-1,491.69)$ \\
Breakfast & $278.10 \pm 124.59(0-960.57)$ \\
Morning snack & $162.92 \pm 99.65(0-640.50)$ \\
Lunch & $399.63 \pm 162.40(0-1,079.55)$ \\
Afternoon snack & $195.57 \pm 100.00(0-637.41)$ \\
Dinner & $454.43 \pm 203.78(0-1,294.26)$ \\
Evening snack & $197.54 \pm 143.91(0-900.19)$ \\
Total number of data entry averages for 6 months & \\
Body weight & $76.50 \pm 56.56(6.00-611.00)$ \\
Exercise & $63.11 \pm 72.59(0-602.00)$ \\
Breakfast & $118.37 \pm 95.03(0-616.00)$ \\
Morning snack & $44.75 \pm 59.29(0-589.00)$ \\
Lunch & $106.80 \pm 90.05(0-596.00)$ \\
Afternoon snack & $53.66 \pm 61.87(0-572.00)$ \\
Dinner & $89.62 \pm 84.38(0-668.00)$ \\
Evening snack & $28.23 \pm 44.64(0-567.00)$ \\
\hline
\end{tabular}

SD, standard deviation.

ing the 6 months (Table 3). In terms of demographic factors, users in the $5 \%$ body weight reduction group were younger $(t(23,680)=$ 910.88, $P<0.001)$ and had higher baseline body weight $\mathrm{t}(23,680)=$ $-4.57, P<0.001)$ and BMI $\left(\chi^{2}(1, \mathrm{~N}=23,682)=12.85, P<0.001\right)$. The two groups also showed significant differences in gender $\left(\chi^{2}(1, \mathrm{~N}=23,682)=54.91, P<0.001\right)$, app version $\left(\chi^{2}(1, \mathrm{~N}=\right.$ $23,682)=4.33, P=0.038)$, location $\left(\chi^{2}(4, \mathrm{~N}=23,682)=119.88\right.$, $P<0.001)$, and average exercise calories $(\mathrm{t}(23,680)=-4.54, P<$ $0.001)$.

Specifically, in comparison to the non-5\% reduction group, the $5 \%$ reduction group at baseline consumed significantly lower amounts of calories for breakfast $(\mathrm{t}(23,680)=12.84, P<0.001)$, morning snack $(\mathrm{t}(23,680)=3.67, P<0.001)$, lunch $(\mathrm{t}(23,680)=$ $4.61, P<0.001)$, and dinner $(\mathrm{t}(23,680)=2.34, P=0.019)$. Furthermore, in comparison to their less effective counterparts, users in the $5 \%$ reduction group burned higher amounts of calories through exercise $(\mathrm{t}(23,680)=-4.54, P<0.001)$. With regard to the total number of data entries for 6 months, users in the $5 \%$ body weight reduction group entered data significantly more frequently for body weight $(\mathrm{t}(23,680)=-13.75, P<0.001)$, exercise $(\mathrm{t}(23,680)=$ $-14.83, P<0.001)$, breakfast $(\mathrm{t}(23,680)=-16.81, P<0.001)$, morning snack $(\mathrm{t}(23,680)=-11.58, P<0.001)$, lunch $(\mathrm{t}(23,680)=$ 
Table 3. Differences in demographic and self-monitoring factors between $5 \%$ and non- $5 \%$ reduction of body weight groups $(n=23,682)$

\begin{tabular}{|c|c|c|c|}
\hline Characteristics & $\begin{array}{l}5 \% \text { Reduction group } \\
(n=15,707,66.3 \%)\end{array}$ & $\begin{array}{l}\text { Non-5\% reduction group } \\
\quad(n=7,975,33.7 \%)\end{array}$ & $P$ \\
\hline \multicolumn{4}{|l|}{ Demographic factor } \\
\hline Age (yr) & $36.00 \pm 10.82$ & $37.64 \pm 11.26$ & $<0.001^{\dagger}$ \\
\hline Baseline body weight $(\mathrm{kg})$ & $90.95 \pm 19.28$ & $89.74 \pm 19.22$ & $<0.001^{\dagger}$ \\
\hline Body mass index $\left(\mathrm{kg} / \mathrm{m}^{2}\right)$ & & & $<0.001^{\dagger}$ \\
\hline Overweight & $7,623(48.50)$ & $4,067(51.00)$ & \\
\hline Obese & $8,084(51.50)$ & $3,908(49.00)$ & \\
\hline Sex & & & $<0.001^{\dagger}$ \\
\hline Female & $10,566(67.30)$ & 5,741 (71.99) & \\
\hline Male & $5,141(32.70)$ & 2,234 (28.01) & \\
\hline App version & & & $0.038^{\dagger}$ \\
\hline Free trial & $14,674(93.42)$ & 7,393 (92.70) & \\
\hline Paid version & $1,033(6.58)$ & $582(7.30)$ & \\
\hline Location* & & & $<0.001^{\dagger}$ \\
\hline Europe & $6,729(42.89)$ & 2,880 (36.14) & \\
\hline America & 5,589 (35.63) & 3,354 (42.08) & \\
\hline Asia & 2,667 (16.98) & $1,345(16.88)$ & \\
\hline Australia & $399(2.50)$ & $225(2.82)$ & \\
\hline Africa & $304(1.94)$ & $166(2.08)$ & \\
\hline \multicolumn{4}{|l|}{ Self-monitoring factor } \\
\hline \multicolumn{4}{|l|}{ Calorie average (kcal) } \\
\hline Exercise & $297.33 \pm 206.76$ & $284.38 \pm 208.44$ & $<0.001^{\dagger}$ \\
\hline Breakfast & $275.24 \pm 122.38$ & $283.74 \pm 128.66$ & $<0.001^{\dagger}$ \\
\hline Morning snack & $161.23 \pm 97.09$ & $166.25 \pm 104.43$ & $<0.001^{\dagger}$ \\
\hline Lunch & $396.17 \pm 157.49$ & $406.45 \pm 171.46$ & $<0.001^{\dagger}$ \\
\hline Afternoon snack & $194.86 \pm 96.89$ & $196.97 \pm 105.85$ & 0.126 \\
\hline Dinner calories & $452.22 \pm 197.37$ & $458.78 \pm 215.80$ & $0.019^{\dagger}$ \\
\hline Evening snack & $197.91 \pm 139.95$ & $196.79 \pm 151.41$ & 0.571 \\
\hline \multicolumn{4}{|c|}{ Total number of data entry averages for 6 months } \\
\hline Body weight & $80.09 \pm 57.76$ & $69.44 \pm 53.41$ & $<0.001^{\dagger}$ \\
\hline Exercise & $68.07 \pm 74.87$ & $53.44 \pm 66.80$ & $<0.001^{\dagger}$ \\
\hline Breakfast & $235.72 \pm 96.24$ & $103.88 \pm 90.87$ & $<0.001^{\dagger}$ \\
\hline Morning snack & $47.92 \pm 61.14$ & $38.51 \pm 54.92$ & $<0.001^{\dagger}$ \\
\hline Lunch & $115.05 \pm 91.43$ & $90.57 \pm 85.04$ & $<0.001^{\dagger}$ \\
\hline Afternoon snack & $58.74 \pm 64.05$ & $43.65 \pm 55.99$ & $<0.001^{\dagger}$ \\
\hline Dinner & $98.06 \pm 86.32$ & $73.00 \pm 77.78$ & $<0.001^{\dagger}$ \\
\hline Evening snack & $31.66 \pm 46.87$ & $21.45 \pm 39.03$ & $<0.001^{\dagger}$ \\
\hline
\end{tabular}

Values are presented as mean \pm standard deviation or number (\%).

*Some values missing; ' Significant level at $P$-value $<0.05$.

-19.94, $P<0.001)$, afternoon snack $(\mathrm{t}(23,680)=-17.86, P<0.001)$, dinner $(\mathrm{t}(23,680)=-21.82, P<0.001)$, and evening snack $(\mathrm{t}(23,680)=$ $-16.74, P<0.001)$. Users who succeed in losing $5 \%$ of their body weight entered more data on their exercise, meals, and body weight. From the results, it can be interpreted that monitoring calorie expenditure and consumption by using the app to regularly and fre-
Table 4. Logistic regression predicting likelihood of reporting $5 \%$ body weight reduction

\begin{tabular}{lccccc}
\hline Variable & $\beta$ & SE & Wald & Sig & Odds ratio (95\% CI) \\
\hline Age & -0.020 & 0.001 & 294.13 & $<0.001$ & $0.976(0.970-0.980)$ \\
Sex & 0.200 & 0.033 & 37.77 & $<0.001$ & $1.226(1.150-1.310)$ \\
Initial BMl & 0.010 & 0.003 & 12.73 & $<0.001$ & $1.009(1.004-1.010)$ \\
$\begin{array}{c}\text { Total number of body weight } \\
\text { data entries for 6 months }\end{array}$ & 0.004 & 0.000 & 127.06 & $<0.001$ & $1.004(1.003-1.005)$ \\
$\begin{array}{c}\text { Total number of exercise } \\
\text { data entries for 6 months }\end{array}$ & 0.002 & 0.000 & 48.49 & $<0.001$ & $1.002(1.001-1.002)$ \\
$\begin{array}{c}\text { Total number of dinner data } \\
\text { entries for 6 months }\end{array}$ & 0.004 & 0.000 & 321.05 & $<0.001$ & $1.004(1.004-1.005)$ \\
\hline
\end{tabular}

SE, standard error; Sig, significance; Cl, confidence interval; BMl, body mass index.

quently enter data has a positive impact on losing body weight.

\section{Factors influencing $\mathbf{5 \%}$ body weight reduction}

Logistic regression was performed to evaluate the impact of a number of factors on the likelihood of $5 \%$ body weight reduction. Based on the results, the final model contains six different factorsage, sex, initial BMI, total body weight, exercise, and dinner data entries during the 6 months. The full model containing all predictors was statistically significant, $\chi^{2}(6, \mathrm{~N}=23,682)=1,124.97, P<$ 0.001 , indicating that the model was able to distinguish between the two groups of users. As shown in Table 4, six variables significantly contributed to the model. The strongest predictor was sex, with an odds ratio (OR) of 1.226. Controlling for all other factors in the model, we found that male users were 1.23 times more likely than female to achieve $5 \%$ body weight reduction ( $95 \%$ confidence interval $[\mathrm{CI}], 1.15-1.31 ; P<0.001)$ across each of the two groups. For every year increase in age, users in each of the two groups were 0.98 times less likely to achieve 5\% body weight reduction (95\% CI, 0.97-0.98; $P<0.001)$. In each of the two groups, we found that users with a higher BMI at baseline were 1.009 times more likely to achieve 5\% body weight reduction (95\% CI, 1.004-1.014; $P<0.001)$. Finally, users who frequently entered data about their body weight (OR, 1.004; 95\% CI, 1.003-1.005; $P<0.001$ ), exercise habits (OR, 1.002; 95\% CI, 1.001-1.002; $P<0.001)$, or dinner (OR, 1.004; 95\% CI, 1.004-1.005; $P<0.001$ ) had a significantly higher probability of achieving $5 \%$ body weight reduction.

\section{Time required to achieve $5 \%$ body weight reduction}

Next, in order to explore the time needed to achieve $5 \%$ body 
A

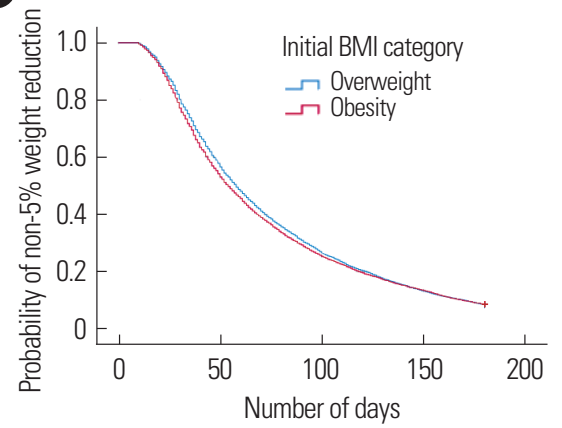

D

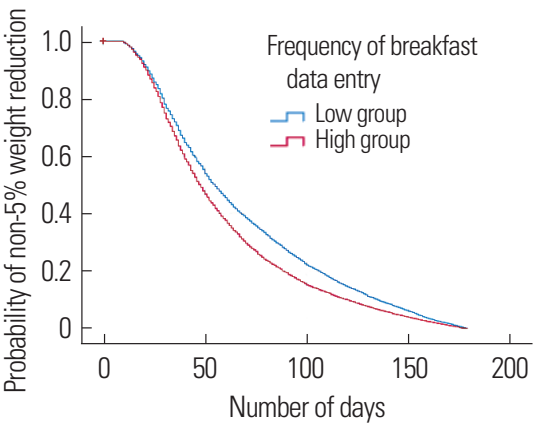

G

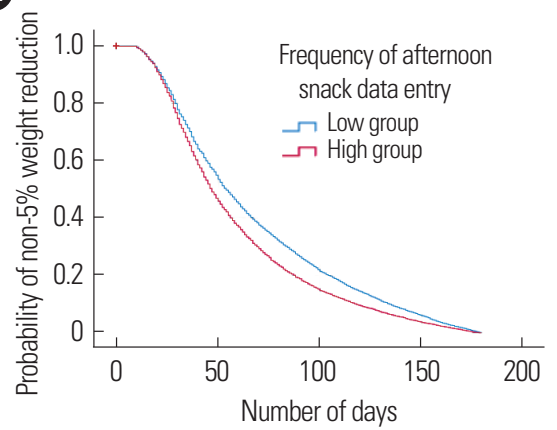

B

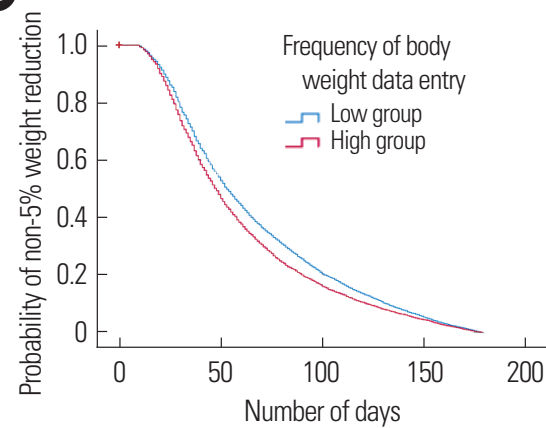

E

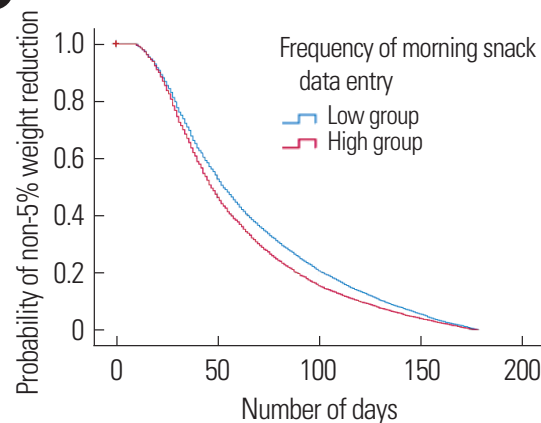

(H)

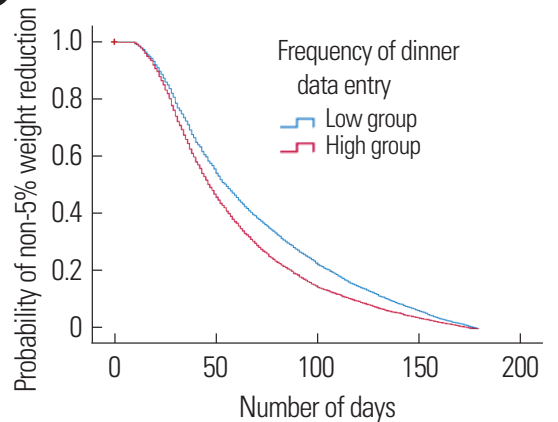

C

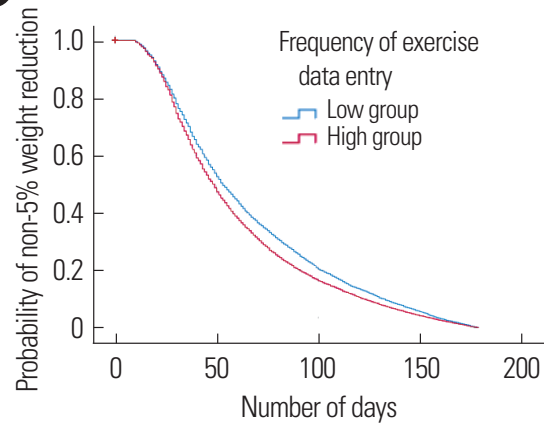

•

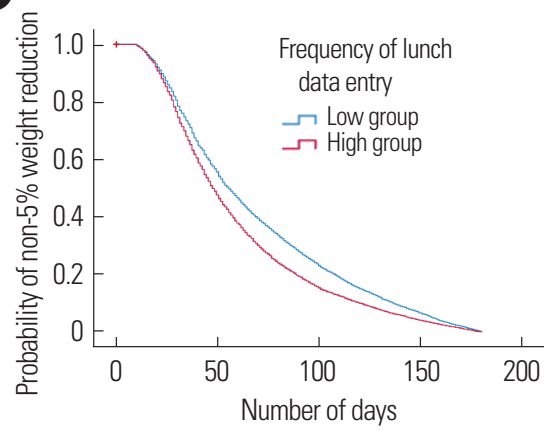

(1)

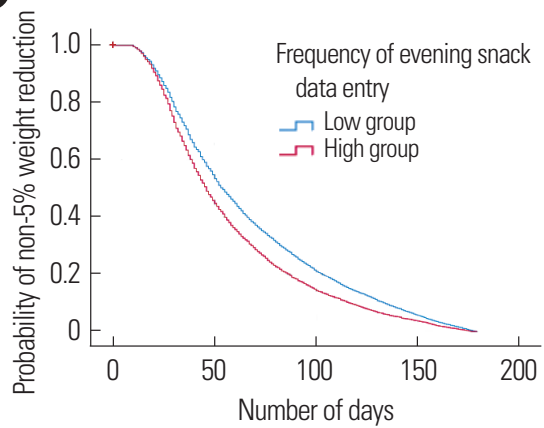

Figure 1. (A) The plot shows that obese users achieved $5 \%$ body weight reduction earlier than overweight users. (B) The plot shows that users in the high data entry group achieved $5 \%$ body weight reduction sooner than users in the low group. (C) The plot shows that users in the high data entry group achieved $5 \%$ body weight reduction sooner than users in the low group. (D) The plot shows that users in the high data entry group achieved $5 \%$ body weight reduction sooner than users in the low group. (E) The plot shows that users in the high data entry group achieved $5 \%$ body weight reduction sooner than users in the low group. (F) The plot shows that users in the high data entry group achieved $5 \%$ body weight reduction sooner than users in the low group. (G) The plot shows that users in the high data entry group achieved $5 \%$ body weight reduction sooner than users in the low group. $(H)$ The plot shows that users in the high data entry group achieved $5 \%$ body weight reduction sooner than users in the low group. (I) The plot shows that users in the high data entry group achieved $5 \%$ body weight reduction sooner than users who in the low group.

weight reduction, we conducted K-M plots and log-rank tests (Fig. 1). The amount of time that users needed to achieve 5\% body weight reduction significantly differed depending on initial BMI and the total number of data entries for body weight, exercise, and dietary intake including breakfast, morning snack, lunch, afternoon snack, dinner, and evening snack information. We found that users who were obese achieved $5 \%$ body weight reduction around 62 days
(95\% CI, 47.95-50.06) of using the app. This is significantly earlier than the time to $5 \%$ body weight reduction for overweight users (65 days: 95\% CI, 51.91-54.09; $P<0.001$ ). Furthermore, users who frequently entered body weight data achieved the $5 \%$ body weight reduction about 3 days faster (62 days: $95 \% \mathrm{CI}, 47.95-50.06)$ than users who entered body weight data less frequently (65 days: $95 \%$ CI, 51.91-54.09; $P<0.001)$. Likewise, users who frequently entered 
their exercise data achieved the $5 \%$ body weight reduction 6 days earlier (60 days: 95\% CI, 46.87-49.13) than users in the low data entry group (66 days: 95\% CI, 51.90-54.10; $P<0.001$ ).

In addition, app users who frequently entered breakfast data were about seven days faster in achieving the $5 \%$ body weight reduction than their counterparts (60 days: 95\% CI, 47.10-48.91 vs. 67 days: 95\% CI, 53.79-56.21; $P<0.001)$. Similar trends were observed with regard to the frequency of data entry about morning snack, lunch, afternoon snack, dinner, and evening snack by app users. Specifically, app users who entered this dietary intake data more frequently achieved the $5 \%$ body weight reduction $6,8,8,9$, and 9 days earlier, respectively, than their counterparts from the low group (66 days: 95\% CI, 52.00-54.00 vs. 60 days: $95 \%$ CI, $45.95-48.05, P<0.001$; 59 days: $95 \%$ CI, 53.78-56.22 vs. 68 days: 95\% CI, 46.09-47.92, $P<0.001$; 59 days: $95 \%$ CI, $46.02-47.98$ vs. 67 days: $95 \%$ CI, $52.92-$ 55.08, $P<0.001$; 59 days: $95 \%$ CI, $46.05-47.95$ vs. 68 days: $95 \% \mathrm{CI}$, 53.83-56.17, $P<0.001$; 58 days: $95 \%$ CI, $44.91-47.09$ vs. 67 days: 95\% CI, 52.97-55.03, $P<0.001$, respectively).

\section{Factors related to time required to achieve 5\% body weight reduction}

Cox hazard regression models were used to evaluate the impact of the different variables that related to the time that users needed to achieve $5 \%$ body weight reduction. The final model was determined (Table 5). The model explains that, with an increase of every unit in initial body weight, successful achievement of $5 \%$ body weight reduction was 1.002 times more likely (adjusted hazard ratio [aHR], 1.002; 95\% CI, 1.001-1.003; $P<0.001)$. In addition, with an increase of every unit in the total number of body weight data entries, successful achievement of $5 \%$ body weight reduction was 1.001 times more likely (aHR, 1.001; 95\% CI, 1.001-1.003; $P<0.001$ ). Similar

Table 5. Factors related to time to $5 \%$ body weight reduction

\begin{tabular}{lcccccc}
\hline Covariate & $\beta$ & SE & Wald & Sig & Exp (B) & $95 \%$ Cl \\
\hline $\begin{array}{l}\text { Initial body weight } \\
\text { Total number of body weight }\end{array}$ & 0.002 & 0.000 & 31.32 & $<0.001$ & 1.002 & $1.001-1.003$ \\
$\begin{array}{c}\text { data entries for 6 months } \\
\text { Total number of dinner data } \\
\text { entries for 6 months }\end{array}$ & 0.000 & 36.21 & $<0.001$ & 1.001 & $1.001-1.003$ \\
$\begin{array}{c}\text { Total number of evening snack } \\
\text { data entries for 6 months }\end{array}$ & 0.001 & 0.000 & 14.01 & $<0.001$ & 1.001 & $1.000-1.001$ \\
\hline
\end{tabular}

SE, standard error; Sig, significance; $\mathrm{Cl}$, confidence interval. increases in the likelihood of achieving $5 \%$ body weight reduction were found for an increase in every unit in the frequency of dinner data entry (1.003 times; aHR, 1.003; 95\% CI, 1.00-1.001; $P<0.001$ ) and dinner data entry (1.001 times; aHR, 1.001; 95\% CI, 1.00 1.001; $P<0.001)$ (Table 5).

\section{DISCUSSION}

As the global epidemic in obesity continues, a recent report shows that about two billion people (i.e., 30\% of the world's population) are overweight or obese. ${ }^{17}$ Previous studies have linked obesity and being overweight to higher risks for health complications, such as type 2 diabetes, cardiovascular diseases, mood disorders, respiratory problems, major cancers, and other health problems. ${ }^{17,18}$ In addition, obesity is associated with higher rates of death driven by comorbidities. $^{19,20}$

In this context, there is evidence that losing $5 \%$ of overall body weight provides health benefits by reducing the risks of some obesity-related diseases in obese people. ${ }^{21,22}$ When obese people lose $5 \%$ of their body weight, their insulin-secreting beta cells improve, as does insulin sensitivity in fat, liver, and skeletal muscle tissue, thereby lowering the risk for diabetes and cardiovascular disease. In addition, total body fat and fatty liver levels also significantly decrease in obese people who achieve $5 \%$ body weight reduction. ${ }^{23}$ In recent years, a variety of tools have been introduced to support successful body weight reduction in obese people. With the extensive market penetration of mobile phones, many studies have identified that mHealth apps are the most promising and effective tools for losing body weight. ${ }^{24}$

This study verifies the significant effects of a popular mHealth app on 5\% body weight reduction in overweight and obese adults for a study period of 6 months. The results demonstrate that about two-thirds of users achieved 5\% body weight loss after using the fitness and weight loss app Noom Coach. In addition, we found that users who were obese were more likely to succeed in the $5 \%$ reduction of body weight.

The percentage of achievement of $5 \%$ body weight reduction among obese mHealth app users was higher herein than values reported in previous studies that have focused on mobile device interventions. For instance, the work of Mao et al. ${ }^{11}$ reports that re- 
search participants lost an average of $3.23 \%$ of total body weight at 4 months of weight loss efforts, while $28.6 \%$ showed a reduction in body weight of $5 \%$ or more after using a mobile phone-based health body weight loss coaching intervention. Likewise, in a study on obese U.S. adults in the general population who reported trying to lose body weight, Nicklas et al. ${ }^{25}$ finds that $40 \%$ of subjects who attempted body weight reduction lost over $5 \%$ of body weight. In comparison to the results of previous studies, the mHealth app intervention evaluated in the present study showed greater effects in the $5 \%$ reduction of body weight among subjects. Due to differences in the interventions and study populations, however, our results and the findings of previous studies should not be frankly compared. Indeed, further research is needed to validate and generalize the effectiveness of the mHealth app intervention evaluated in the present study.

Our results demonstrated that younger users were more likely to attain 5\% body weight reduction with usage of the mHealth app. Furthermore, we found that those who frequently monitored their body weight, exercise habits, and dietary intake were more likely to succeed in losing 5\% body weight in comparison to users who entered less data into the app. It can be assumed that people come to understand their eating habits and exercise patterns though the regular entry of data into the app. This monitoring process allows users to lose weight by balancing calories consumed from food and expended by exercise throughout the day.

In line with the results of this study, the work of Carroll et al. ${ }^{26}$ shows that mobile phone ownership is higher among younger people and is associated with an increased likelihood of having adopted health apps and having received health benefits by using the apps. Without a specific focus on 5\% body weight reduction, research by Turner-Mcgrievy et al. ${ }^{27}$ finds that users who self-monitored exercise more frequently for 6 months significantly reduced their BMI. In that work, however, frequency in diet self-monitoring did not show the same effectiveness in changes in $\mathrm{BMI} .{ }^{27}$ In contrast, the work of Laing et al. ${ }^{28}$ finds no significant effectiveness in the use of self-monitoring of dietary intake and physical activity with $\mathrm{mHealth}$ apps for body weight changes. Likewise, Peterson et al. ${ }^{29}$ find no impact of total frequency of dietary intake self-monitoring on body weight reduction. Additionally, a randomized controlled trial reports no effectiveness of mobile app-based self-monitoring of physical and dietary intake in body weight changes among participants at 3 months. ${ }^{30}$ Previous findings on the effectiveness of self-monitoring on body weight changes by way of mHealth app usage are inconsistent. Different types of mHealth apps, different study populations, and various study periods might impact these different results. Therefore, more randomized controlled trials are needed to investigate the effects of self-monitoring on $5 \%$ body weight reduction through mHealth apps, as well as to accurately determine which self-monitoring content is useful for $5 \%$ body weight reduction.

According to our results, it took overweight or obese app users 81 days (about 2.5 months) to reduce their original body weight by $5 \%$. This finding suggests that the mHealth app analyzed in the present study has a positive impact on 5\% body weight reduction in a shorter time than has been established in previous studies in different types of interventions. From these results, it can be assumed that monitoring diet, exercise habits, and/or weight can increase people's perceptions of the effects of behavior modification on weight reduction. In order to enter their body weight data into the app, users would have had to check their body weight regularly. Body weight monitoring is a simple and crucial way to reduce weight by boosting motivation. Regular monitoring also allows users to notice and record exercise calories burned throughout the day, thus encouraging more movement. In this way, people can efficiently reduce their body weight in a short period of time. ${ }^{31}$

Research by Torgan ${ }^{32}$ reports that 19 participants achieved an initial target of $5 \%$ body weight reduction after approximately 3.5 months of intervention consisting of a low-calorie diet and weekly diet and education sessions. Based on these findings, it can be concluded that mHealth apps are an innovative solution to decreasing body weight in a short period of time for obese populations. ${ }^{33}$ However, it is difficult to conclude that body weight reduction in a short period of time is a positive result, because the ideal goal and time to body weight reduction might vary depending on the health status of users. In addition, there is no certainty as to whether body weight reduction is caused by the loss of body fat, muscle, or water weight. Therefore, this study recommends against considering only changes in body weight as an outcome variable, and instead examining changes in body composition of fat and muscle and/or health outcomes such as blood pressure, glucose, or cholesterol levels as additional outcome variables. Because relevant 
research on this topic is rare, future well-structured randomized studies are needed to examine the effectiveness of mHealth apps in not only $5 \%$ body weight reduction, but also with regard to changing body composition in overweight or obese mHealth app users.

\section{Strengths and limitations}

The present study is the first to examine the effects of using a popular mHealth app in overweight and obese adults to achieve 5\% body weight reduction. Our results contribute to existing evidence that mHealth apps are affordable and easy to use in reducing body weight by $5 \%$, especially in overweight or obese users. The results of this study might have a high level of generalizability due to our large-scale, international dataset. This study demonstrates that mobile app-based coaching intervention is an acceptable and effective tool in achieving recommended body weight loss for overweight and obese individuals within a short period of time.

The present study has several limitations. First, our results are limited because of the study design of secondary data analysis. Therefore, well-designed randomized controlled trials are needed to carefully examine the effectiveness of mHealth apps in 5\% body weight loss in overweight or obese users. Second, our data did not include some demographic characteristics (e.g., education level, income level, health literacy, and health status) that might have impacted body weight changes in subjects. Third, the use of self-reported data may be problematic in terms of accurate evaluation of intervention effectiveness. Fourth, this study excluded data from users aged 42 years because 42 years is the default age value of the mHealth app. Insofar as data from actual 42-year-old users might have been excluded from analysis, our findings were potentially impacted by data omission. Finally, it remains unclear from our results whether our study population used only the app to lose body weight or whether they also pursued other activities such as using the gym, meditation, personal training, and/or diet therapy.

\section{Conclusion}

Based on the results of this study, it can be concluded that mHealth apps are promising and effective tools to achieve 5\% body weight reduction in overweight and obese adults within a relatively short period of time. Specifically, users who frequently self-monitor their weight loss behaviors by regularly entering data into mHealth apps show greater effects in body weight loss with a shorter time required to achieve the weight loss goal.

\section{CONFLICTS OF INTEREST}

The authors declare no conflict of interest.

\section{ACKNOWLEDGMENTS}

This study was supported by a grant from the Korean Health Technology R\&D Project, Ministry of Health and Welfare, Republic of Korea (HI16C2048).

The abstract of this manuscript was presented as a poster at the 53rd Korean Society for the Study of Obesity (KSSO) annual spring conference. The poster was awarded with recognition as an excellent poster.

The authors would like to thank Prof. Emeritus Young Seol Kim from Kyung Hee University for his exceptional teaching and inspiration.

\section{AUTHOR CONTRIBUTIONS}

Study concept and design: MH; acquisition of data: SYR; analysis and interpretation of data: $\mathrm{MH}$; drafting of the manuscript: all authors; critical revision of the manuscript: all authors; statistical analysis: $\mathrm{MH}$; obtained funding: SYR; administrative, technical, and material support: SYR; and study supervision: SYR.

\section{REFERENCES}

1. World Health Organization. Obesity and overweight [Internet]. Geneva: World Health Organization; 2021 [cited 2021 Jan 18]. Available from: https://www.who.int/news-room/fact-sheets/ detail/obesity-and-overweight

2. WebMD. Health risks linked to obesity [Internet]. New York (NY): WebMD; 2017 [cited 2021 Jan 18]. Available from: https://www.webmd.com/diet/obesity/obesity-health-risks\#1

3. Gorin A. 7 Reasons to lose 5 percent of your body weight [Internet]. New York (NY): Everyday Health; 2016 [cited 2021 Jan 18]. Available from: https://www.everydayhealth. 
com/news/reasons-lose-5-percent-body-weight/

4. Centers for Disease Control and Prevention. Losing weight [Internet]. Atlanta (GA): Centers for Disease Control and Prevention; 2018 [cited 2021 Jan 18]. Available from: https:// www.cdc.gov/healthyweight/losing weight/index.html

5. Apovian CM, Garvey WT, Ryan DH. Challenging obesity: patient, provider, and expert perspectives on the roles of available and emerging nonsurgical therapies. Obesity (Silver Spring) 2015;23 Suppl 2:S1-26.

6. Montesi L, El Ghoch M, Brodosi L, Calugi S, Marchesini G, Dalle Grave R. Long-term weight loss maintenance for obesity: a multidisciplinary approach. Diabetes Metab Syndr Obes 2016;9:37-46.

7. Madigan CD, Pavey T, Daley AJ, Jolly K, Brown WJ. Is weight cycling associated with adverse health outcomes? A cohort study. Prev Med 2018;108:47-52.

8. Zhao J, Freeman B, Li M. Can mobile phone apps influence people's health behavior change?: an evidence review. J Med Internet Res 2016;18:e287.

9. Brindal E, Hendrie G, Freyne J, Coombe M, Berkovsky S, Noakes M. Design and pilot results of a mobile phone weight-loss application for women starting a meal replacement programme. J Telemed Telecare 2013;19:166-74.

10. Levitsky DA, Garay J, Nausbaum M, Neighbors L, Dellavalle DM. Monitoring weight daily blocks the freshman weight gain: a model for combating the epidemic of obesity. Int J Obes (Lond) 2006;30:1003-10.

11. Mao AY, Chen C, Magana C, Caballero Barajas K, Olayiwola JN. A mobile phone-based health coaching intervention for weight loss and blood pressure reduction in a national payer population: a retrospective study. JMIR Mhealth Uhealth 2017; 5:e80.

12. Pagoto S, Schneider K, Jojic M, DeBiasse M, Mann D. Evidencebased strategies in weight-loss mobile apps. Am J Prev Med 2013;45:576-82.

13. Chin SO, Keum C, Woo J, Park J, Choi HJ, Woo JT, et al. Successful weight reduction and maintenance by using a smartphone application in those with overweight and obesity. Sci Rep 2016;6:34563.

14. Ustulin M, Keum C, Woo J, Woo JT, Rhee SY. Effects of cli- matic variables on weight loss: a global analysis. Sci Rep 2017; $7: 40708$

15. Centers for Disease Control and Prevention. Body mass index (BMI) [Internet]. Atlanta (GA): Centers for Disease Control and Prevention; 2021 [cited 2021 Jan 18]. Available from: https://www.cdc.gov/healthyweight/assessing/bmi/index. html

16. Centers for Disease Control and Prevention. Defining adult overweight and obesity [Internet]. Atlanta (GA): Centers for Disease Control and Prevention; 2021 [cited 2021 Jan 18]. Available from: https://www.cdc.gov/obesity/adult/defining. html

17. Sifferlin A. Almost $30 \%$ of people in the world are obese or overweight [Internet]. New York (NY): Time; 2017 [cited 2021 Jan 18]. Available from: https://time.com/4813075/ obesity-overweight-weight-loss/

18. Haslam DW, James WP. Obesity. Lancet 2005;366:1197-209.

19. Abdelaal M, le Roux CW, Docherty NG. Morbidity and mortality associated with obesity. Ann Transl Med 2017;5:161.

20. Jarolimova J, Tagoni J, Stern TA. Obesity: its epidemiology, comorbidities, and management. Prim Care Companion CNS Disord 2013; 15:PCC.12f01475.

21. Dockrill P. The first 5\% of weight loss offers the greatest health benefits in obese people, study finds [Internet]. Canberra: ScienceAlert; 2016 [cited 2021 Jan 18]. Available from: https:// www.sciencealert.com/the-first-5-of-weight-loss-offers-thegreatest-health-benefits-in-obese-people-study-finds

22. Wing RR, Lang W, Wadden TA, Safford M, Knowler WC, Bertoni AG, et al. Benefits of modest weight loss in improving cardiovascular risk factors in overweight and obese individuals with type 2 diabetes. Diabetes Care 2011;34:1481-6.

23. Magkos F, Fraterrigo G, Yoshino J, Luecking C, Kirbach K, Kelly SC, et al. Effects of moderate and subsequent progressive weight loss on metabolic function and adipose tissue biology in humans with obesity. Cell Metab 2016;23:591-601.

24. Flores Mateo G, Granado-Font E, Ferré-Grau C, MontañaCarreras X. Mobile phone Apps to promote weight loss and increase physical activity: a systematic review and meta-analysis. J Med Internet Res 2015;17:e253.

25. Nicklas JM, Huskey KW, Davis RB, Wee CC. Successful weight 
loss among obese U.S. adults. Am J Prev Med 2012;42:481-5.

26. Carroll JK, Moorhead A, Bond R, LeBlanc WG, Petrella RJ, Fiscella K. Who uses mobile phone health apps and does use matter?: a secondary data analytics approach. J Med Internet Res 2017;19:e125.

27. Turner-McGrievy GM, Beets MW, Moore JB, Kaczynski AT, Barr-Anderson DJ, Tate DF. Comparison of traditional versus mobile app self-monitoring of physical activity and dietary intake among overweight adults participating in an mHealth weight loss program. J Am Med Inform Assoc 2013;20:513-8.

28. Laing BY, Mangione CM, Tseng CH, Leng M, Vaisberg E, Mahida M, et al. Effectiveness of a smartphone application for weight loss compared with usual care in overweight primary care patients: a randomized, controlled trial. Ann Intern Med 2014;161(10 Suppl):S5-12.

29. Peterson ND, Middleton KR, Nackers LM, Medina KE, Milsom VA, Perri MG. Dietary self-monitoring and long-term success with weight management. Obesity (Silver Spring) 2014;22:1962-7.
30. Patel ML, Hopkins CM, Brooks TL, Bennett GG. Comparing self-monitoring strategies for weight loss in a smartphone App: randomized controlled trial. JMIR Mhealth Uhealth 2019;7: e12209.

31. Han M, Rhee SY. Effect of adherence to smartphone app use on the long-term effectiveness of weight loss in developing and OECD countries: retrospective cohort study. JMIR Mhealth Uhealth 2021;9:e13496.

32. Torgan C. NIH research matters: benefits of moderate weight loss in people with obesity [Internet]. Bethesda (ML): National Institutes of Health; 2016 [cited 2021 Jan 18]. Available from: https://www.nih.gov/news-events/nih-research-matters/benefits-moderate-weight-loss-people-obesity

33. Beaton T. mHealth apps, patient coaching improve weight loss, heart health [Internet]. Thinkstock; 2017 [cited 2021 Jan 18]. Available from: https://mhealthintelligence.com/ news/mhealth-apps-patient-coaching-improve-weight-lossheart-health 